\title{
Bogijetong decoction and its active herbal components protect the peripheral nerve from damage caused by taxol or nerve crush
}

So Hyun Ahn, In Ae Chang, Ki-Joong Kim, Chul-Jung Kim, Uk Namgung and Chung-Sik Cho*

\begin{abstract}
Background: Bogijetong decoction (BGJTD) is a herbal drug formulation used in the traditional Asian medicine to treat neuropathic insults associated with diabetes and anticancer therapy. To understand the biological basis of BGJTD on protective effects against neuropathy, we investigated physiological and biochemical responses of the sciatic nerves deranged by taxol injection or crush injury in the rats.

Methods: Dissociated Schwann cells and neurons were prepared from the sciatic nerve and dorsal root ganglia (DRG) respectively and were treated with taxol and BGJTD. The sciatic nerve in the rat was injected with taxol or given crush injury. Animals were then administered orally with BGJTD. Effects of BGJTD treatment on cultured cells and in vivo sciatic nerves and DRG tissues were examined by immunofluorescence staining and western blot analysis. Sciatic nerve regeneration was assessed by histological observation using retrograde tracing technique and by behavioral hot plate test. Eighteen different herbal components of BGJTD were divided into 4 subgroups and were used to select herbal drugs that enhanced neurite outgrowth in cultured neurons.

Results: Morphological abnormalities in the sciatic nerve axons and DRG tissue caused by taxol injection were largely improved by BGJTD treatment. BGJTD treatment enhanced neurite outgrowth in cultured DRG neurons and improved Schwann cell survival. Phospho-Erk1/2 levels were elevated by BGJTD administration in the injured- or taxol-injected sciatic nerves. Vimentin phosphorylation catalyzed by cell division cycle 2 (Cdc2) kinase was induced from Schwann cells in the sciatic nerves after taxol injection and crush injury, and phospho-vimentin levels were further upregulated by BGJTD treatment. Retrograde tracing of Dil-labeled DRG sensory neurons revealed growth-promoting activity of BGJTD on axonal regeneration. A drug group (Be) composed of 4 active herbal components which were selected by neurite growth-enhancing activity was as effective as BGJDT for the recovery of thermal sensitivity of the hind paws which had been suppressed by taxol administration.
\end{abstract}

Conclusions: These data suggest that BGJTD and its active herbal components may protects the peripheral nerve from damage caused by taxol injection and nerve crush.

Keywords: Bogijetong-decoction, Taxol, Nerve injury, Regeneration, Neuropathy

* Correspondence: choo1o2@dju.k

Department of Oriental Medicine, Daejeon University, Daejeon 300-716,

South Korea International License (http://creativecommons.org/licenses/by/4.0/), which permits unrestricted use, distribution, and reproduction in any medium, provided you give appropriate credit to the original author(s) and the source, provide a link to the Creative Commons license, and indicate if changes were made. The Creative Commons Public Domain Dedication waiver (http://creativecommons.org/publicdomain/zero/1.0/) applies to the data made available in this article, unless otherwise stated. 


\section{Background}

Peripheral neuropathy, referring to histological and physiological abnormalities caused by infections, nerve damages, diabetes, anticancer therapy and others [1], generates deficits of sensory and motor functions and affects the quality of life significantly. At cellular levels, structural alterations in peripheral axons and Schwann cells may be related with deficits in myelination and axonal transport that lead to abnormal conduction property of action potential through the axon [2].

Taxol, known to stabilize microtubule assembly, prevents dynamic regulation of spindle components during mitosis and thus has been used as anticancer therapeutic agent [3]. Yet, clinical studies reported that repeated administration of taxol can generate peripheral neuropathy by disrupting microtubule structure in axons [4-6]. Addition of taxol to cultured neurons resulted in decreased neurite outgrowth, and in vivo administration of taxol in rats generated morphological changes of myelinated fibers and degeneration of Schwann cells [7, 8]. Other studies indicated morphometric alterations via abnormal action potential propagation [9]. Interestingly however, in vivo application of taxol at low dose was shown to improve axonal regeneration after spinal cord injury, by stabilizing microtubule structure and reducing glial scarring around the injury cavity [10].

Bogijetong decoction (or Bogijetong-tang; BGJTD) is the herbal prescription that was developed to reduce neuropathy caused by diabetes and anticancer therapy $[11,12]$. In our previous studies, BGJTD treatment into the streptozotocin (STZ)-induced diabetes animal model induced Schwann cell responses in terms of increased phosphorylation of vimentin by cell division cycle 2 (Cdc2) and induction of phospho-Erk1/2 and $\beta 1$ integrin proteins [13]. Also in an animal model given both sciatic nerve injury and taxol treatments, in vivo administration of BGJDT improved the regenerative responses of injured axons in addition to Schwann cell activation as occurred similarly in STZ-injected animals [14]. While these studies suggest that BGJTD may play a role in regulating peripheral neuropathy, overall neuropathic responsiveness to STZ-injected animals is indirect, and the neuropathy caused by combined physical and chemical paradigm may complicate the interpretation of neural responses to BGJTD. Here, to understand the mechanistic basis on how BGJTD is beneficiary for alleviating neuropathy, we investigated the effects of BGJTD treatment on the sciatic nerve which had been either injected taxol or given crush injury. By examining morphological and biochemical responses of peripheral neurons and Schwann cells, we found that BGJTD has a protective function of peripheral neural tissues against neuropathic insults. Noting that BGJTD is composed of as many as 18 different herbal components, we performed additional experiment to select active components that could represent the efficacy of BGJTD. A small decoction group (Be) composed of 4 herbal components showed a similar efficacy in thermal sensitivity of the hind paw as BGJTD administration.

\section{Methods \\ Herbal drug extraction}

Dried BGJTD was obtained from Daejeon University Korean Hospital (Daejeon, Korea) where the quality control of herbal drugs was in accordance with the Standard of Korean Pharmacopoeia (ver. 9, Korea Food and Drug Administration, Korea). The procedure of identification of 18 individual drugs was executed by Dr. Chung-Sik Cho at Daejeon University Oriental Hospital (Daejeon, Korea). The voucher specimens were deposited in the Herbarium of Pharmacognosy, Department of Oriental Medicine, Daejeon University (Daejeon, Korea). The key information in herbal drugs is summarized in Table 1. BGJTD was used clinically at Korean Traditional Medicine Hospital of Daejeon University (Daejeon, Korea) and its efficacy was verified $[11,12]$. A mixture of dried BGJT was suspended in distilled water for $2 \mathrm{~h}$, boiled for $3 \mathrm{~h}$, and filtered with Whatman filter paper (Grade 1, Whatman Inc, Clifton, NJ, USA) three times. The extract was frozen at $-70{ }^{\circ} \mathrm{C}$ for $4 \mathrm{~h}$ and freeze-dried for $24 \mathrm{~h}$. The yield for BGJTD extract was $24 \mathrm{~g}$ for $178 \mathrm{~g}$ of the initial raw materials. Purified material was stored at $-20{ }^{\circ} \mathrm{C}$ and used for experiment after diluting with physiological saline solution $(1 \mathrm{mg}$ of extract residue $/ \mathrm{ml}$ in $0.9 \% \mathrm{NaCl}$ solution). For the experiment screening the active components, individual herbal drugs of BGJTD were divided into 4 decoction groups according to the traditional medicinal theory (Table 2). Herbal extracts were prepared similarly as above.

\section{HPLC analysis}

Herbal extracts of BGJTD and of $\mathrm{Ba}, \mathrm{Bb}, \mathrm{Bc}, \mathrm{Bd}$, and Be subgroups were purified by filtration through $0.45 \mu \mathrm{m}$ PVDF membrane, and standard solutions such as amygdalin, chlorogenic acid, ginsenosides Rg1 and $\mathrm{Rb} 1$, nodakenin, albiflorin, paeoniflorin, puerarin, and 5-HMF were diluted with ultra-purified water to a range of $50-500 \mathrm{ppm}$. The herbal extracts and standard solutions were analyzed by LC20A Series HPLC system (Shimadzu, Kyoto, Japan). The analysis system includes an Agilent eclipse plus C18 (250x4.6 mm) with linear elution with acetonitrile gradient at a flow rate of $1.0 \mathrm{ml} / \mathrm{min}$. Sample aliquots $(10 \mu \mathrm{l})$ were injected to the column, which was set to $40{ }^{\circ} \mathrm{C}$. UV values for detection were $215 \mathrm{~nm}$ for amygdalin, $203 \mathrm{~nm}$ for $\mathrm{Rg} 1$ and $\mathrm{Rb} 1,230 \mathrm{~nm}$ for puerarin, albiflorin and paeniflorin, $280 \mathrm{~nm}$ for 5-HMF and chlorogenic acid, and $330 \mathrm{~nm}$ for nodakenin. 
Table 1 Sources of BGJTD

\begin{tabular}{llll}
\hline Scientific name & Part & Production area & Specimen number \\
\hline Astragalus membranaceus & Root & Jeongseon, ROK ${ }^{\text {a }}$ & KNP0011 \\
Panax ginseng C. A. Meyer & Root & Geumsan, ROK & KNP0038 \\
Angelica gigas & Root & Jeongseon, ROK & KNP0008 \\
Rehmannia glutinosa & Rhizome & Geumsan, ROK & KNP0087 \\
Cnidium officinale Makino & Rhizome & Younghae, ROK & KNP0028 \\
Paeonia lactiflora Pall & Root & Younghae, ROK & KNP0085 \\
Salvia miltiorrhiza & Root, rhizome & Sichuan, China & KNP0095 \\
Prunus persica & Seed & Hebei, China & KNP0087 \\
Carthamus tinctorius & Flower & Xingang, Taiwan & KNP0020 \\
Spatholobus suberectus Dunn & Stem & Jeongseon, ROK & KNP0102 \\
Epimedium Koreanum NAKAl & Whole & Cheorwon, ROK & KNP0030 \\
Lumbricidae & Whole & Jiangxi, China & KNP0053 \\
Pueraria thunbergiana & Root & Younghae, ROK & KNP0089 \\
Pteridium aquilinum var. latiusculum & Rhizome & Guangdong, China & KNP0023 \\
Albizia julibrissin Durazz & Peel & Gyeongju, ROK & KNP0007 \\
Uncaria rhynchophylla & Branch & Hunan, China & KNP0120 \\
Chaenomeles sinensis & Fruit & Yeongcheon, ROK & KNP0022 \\
Crassostrea gigas & Shell & Mokpo, ROK & KNP0074 \\
Total amount & & & 7.5 \\
\hline
\end{tabular}

${ }^{\mathrm{a}}$ Republic of Korea

Table 2 Subgroups of BGJTD

\begin{tabular}{|c|c|c|}
\hline Subgroups & Composition $^{a}$ & Applications \\
\hline $\mathrm{Ba}$ & $\begin{array}{l}\text { Astragalus membranaceus (30), } \\
\text { Panax ginseng C. A.Meyer } \\
\text { (4, ginsenoside Rg1, Rb1) }^{\mathrm{b}} \\
\text { Epimedium Koreanum NAKAl (10), } \\
\text { Cibotium barometz J. Smith (8) }\end{array}$ & $\begin{array}{l}\text { Replenishing qi and } \\
\text { yin energy }\end{array}$ \\
\hline $\mathrm{Bb}$ & $\begin{array}{l}\text { Angelica gigas (7.5, chlorogenic } \\
\text { acid, nodakenin), Rehmannia } \\
\text { glutinosa (7.5, 5-HMF), Cnidium } \\
\text { officinale Makino (7.5), } \\
\text { Spatholobus suberectus Dunn (12) }\end{array}$ & $\begin{array}{l}\text { Increasing erythropoietic } \\
\text { activity and blood function }\end{array}$ \\
\hline $\mathrm{BC}$ & $\begin{array}{l}\text { Prunus persica ( } 7.5 \text {, amygdaline), } \\
\text { Paeonia lactiflora Pall } \\
\text { (7.5, albiflorin, paeoniflorin), } \\
\text { Carthamus tinctorius (7.5), } \\
\text { Lumbricidae (5), Salvia } \\
\text { miltiorrhiza (12) }\end{array}$ & $\begin{array}{l}\text { Improving blood } \\
\text { circulation }\end{array}$ \\
\hline $\mathrm{Bd}$ & $\begin{array}{l}\text { Uncaria rhynchophylla (12), } \\
\text { Pueraria lobate Ohwi } \\
(8, \text { puerarin), Crassostrea gigas } \\
\text { (12), Albizia julibrissin Durazz (12), } \\
\text { Chaenomeles sinensis Koebhne (8) }\end{array}$ & $\begin{array}{l}\text { Relieving pain and } \\
\text { regulating yang energy }\end{array}$ \\
\hline $\mathrm{Be}$ & $\begin{array}{l}\text { Panax ginseng C. A.Meyer } \\
\text { (4, ginsenoside Rg1, Rb1), } \\
\text { Angelica gigas (7.5), Paeonia } \\
\text { lactiflora Pall (7.5, albiflorin, } \\
\text { paeoniflorin), Crassostrea gigas (8) }\end{array}$ & \\
\hline
\end{tabular}

${ }^{a}$ Number in parenthesis: grams in preparing each decoction

bunderlined in parenthesis are chemical ingredients which were identified by HPLC analysis

\section{Experimental animals}

Sprague-Dawley rats (male, 200-250 g, Samtago, Seoul, Korea) or Balb/c mice (male, 22-25 g, Samtago, Seoul, Korea) were maintained in an animal room with regulated temperature $\left(22{ }^{\circ} \mathrm{C}\right), 60 \%$ of humidity, and 12 -h light/dark cycle (light on 7 am to $7 \mathrm{pm}$ ). They were allowed to eat commercial pellet chow (Samyang Co., Seoul, Korea) and drink water ad libitum. All procedures were in strict accordance with the NIH guide for the care and use of laboratory animals and approved by the Committee on Use of Live Animals for Teaching and Research at Daejeon University (Daejeon, Korea).

\section{Animal surgery}

For an experiment examining the effects of BGJTD in taxol-treated sciatic nerve, animals were randomly assigned into dimethysulfoxide (DMSO) vehicle-injected group (Veh), taxol-injected group (Taxol), and taxol plus BGJTD treated group (Taxol + BGJTD). Sciatic nerves and DRG at levels of lumbar 4 and 5 were prepared from individual animals and used for immunofluorescence staining and western blot analysis. Rats were anesthetized by injecting intraperitoneally a single dose of a mixture of ketamine $(80 \mathrm{mg} / \mathrm{kg})$ and xylazine $(5 \mathrm{mg} / \mathrm{kg})$. Taxol $(3.6 \mathrm{mg} / \mathrm{ml}$ of $5 \%$ DMSO; $1.25 \mathrm{mg} / \mathrm{kg}$ of body weight) and equivalent volume of DMSO as vehicle control were slowly injected by using a Hamilton syringe (10 $\mu$ l-model, Innovative Labor System GmbH, Germany) 
into the sciatic nerve pre-exposed on the middle thigh. A similar procedure of local injection of taxol into the peripheral nerves were used in previous studies to examine regenerative or degenerative effects of taxol on the nerve $[15,16]$. In order to induce acute effect on the microtubule integrity within axon of the peripheral nerve, we injected taxol with higher dosage than those used to investigate the regenerative responses of peripheral and spinal cord axons after injury $[10,16]$. Twenty four hours later, BGJTD $(400 \mathrm{mg} / \mathrm{kg}$ ) was administered orally and was supplemented on a daily basis for 4 more days. Animals were sacrificed at day 7 .

In an experiment investigating the effects of BGJTD on regenerative responses of injured axons, animals were randomly assigned into three groups: sciatic nerve injury plus BGJTD treatment (SNI + BGJTD), sciatic nerve injury plus saline $(\mathrm{SNI}+\mathrm{Sal})$, and non-treatment (Intact). To induce nerve injury, the sciatic nerve was exposed from the middle thigh and crush injury was given by holding the nerve with the forceps for $30 \mathrm{~s}$ twice as described previously [17]. BGJTD or saline was administered orally $24 \mathrm{~h}$ later and supplemented on a daily basis for 4 more days. Animals were sacrificed at day 7 , and sciatic nerve or DRG sections (20 $\mu \mathrm{m}$ thickness) were prepared.

\section{Retrograde tracing of DRG neurons}

For retrograde tracing of the sensory neurons in the DRG, the sciatic nerve from anesthetized rats with ketamine and xylazine was exposed and, immediately after nerve injury, fluorescent lipophilic carbocyanine dye 1,1'-dioctodecyl-3,3,3',3'tetramethylindocarbocyanine perchlorate (DiI; $5 \mu \mathrm{l}$ of $0.5 \%$ in DMSO) was applied with a micropipette to the area $10 \mathrm{~mm}$ distal to the injury site. After suturing the incision, animals were recovered from the narcosis and returned to their cages. BGJTD or saline was administered orally $24 \mathrm{~h}$ later and supplemented on a daily basis for 4 more days, and the animals were sacrificed at day 7 . The total number of DiIlabeled neurons from three nonconsecutive sections (20 $\mu \mathrm{m}$ thick) per animal was counted, and the mean percentage of labeled cells was compared between BGJTD and saline administered groups.

\section{Primary Schwann cell and DRG sensory neuron culture}

For Schwann cell culture, intact sciatic nerve $(1 \mathrm{~cm}$ length) was dissected from the rats and dissociated with $0.5 \mathrm{mg} / \mathrm{ml}$ type XI collagenase (Sigma, USA) in BME for $90 \mathrm{~min}$ at $37{ }^{\circ} \mathrm{C}$. DRG neurons at lumbar $4-5$ in adult rats were prepared 3 days after sciatic nerve injury, dissected, and dissociated similarly for neuron culture. After washing twice with BME, cells were treated with $5 \mu \mathrm{g} / \mathrm{ml}$ type SII trypsin for $15 \mathrm{~min}$ and followed by inhibition reaction for $5 \mathrm{~min}$ in $50 \mu \mathrm{g} / \mathrm{ml}$ of soybean trypsin inhibitor, $0.5 \mathrm{mM}$ EDTA, and $20 \mu \mathrm{g} / \mathrm{ml}$ of DNase
I. Cells $\left(1 \times 10^{4}\right.$ cells per 24-well plate) were plated onto $12 \mathrm{~mm}$ coverslips (Bellco, Glass Inc. Vineland, USA) precoated with $0.01 \%$ poly-L-ornithine (Sigma, St. Louis, MO) and laminin (0.02 $\mathrm{mg} / \mathrm{ml}$, Collaborative Research, Bedford, MA). Cells were cultured for $12 \mathrm{~h}$, changed to BME containing $10 \%$ serum (5\% fetal bovine serum plus $5 \%$ horse serum) and $2 \mathrm{mM}$ glutamine and $1 \%$ penicillin-streptomycin. Cells were treated with herbal drugs $(0.5 \mathrm{mg} / \mathrm{ml})$ and incubated for $48 \mathrm{~h}$ before the harvest for immunofluorescence staining.

For DRG sensory neuron and Schwann cell co-culture, Schwann cells $\left(1 \times 10^{4}\right.$ cells per $12 \mathrm{~mm}$ coverslip in 24-well plate) were cultured for $24 \mathrm{~h}$ and DRG sensory neurons $\left(1.5 \times 10^{2}\right.$ cells $)$ were added. The coculture was maintained in $500 \mu \mathrm{l}$ of BME medium supplemented with $10 \%$ serum and treated with taxol $(0.01 \mathrm{mg} / \mathrm{ml})$ alone or together with BGJTD $(0.5 \mathrm{mg} / \mathrm{ml})$ for $48 \mathrm{~h}$ prior to cell harvest. Equivalent volume of DMSO and saline as vehicle controls for taxol and BGJTD was treated, respectively. After immunofluorescence staining with antibodies against neurofilament-200 (NF-200) (1:400, Sigma), $\beta$ III-tubulin (TUJ1, 1:400, Covance), S100 $\beta$ (H-56, 1:400, Santa Cruz Biotech.), and caspase 3 (1:500, Cell Signaling), digital images of neuronal process were captured and transferred to the Adobe Photoshop Program. The length of neurite processes exhibiting clear outgrowth (longer than cell diameter) from the cell body was analyzed by i-Solution software program (Image and Microscope Technology, Burnaby, Canada). Mean neurite length was determined by analyzing at least 30 sensory neurons which were randomly selected in each experiment.

\section{Western blot analysis}

Nerve segment was suspended in 100-200 $\mu$ l of triton lysis buffer (20 mM Tris, pH 7.4, $137 \mathrm{mM} \mathrm{NaCl}, 25 \mathrm{mM}$ $\beta$-glycerophosphate, $\mathrm{pH} 7.14,2 \mathrm{mM}$ sodium pyrophosphate, $2 \mathrm{mM}$ EDTA, $1 \mathrm{mM} \mathrm{Na} \mathrm{VO}_{4}, 1 \%$ Triton X-100, $10 \%$ glycerol, $5 \mu \mathrm{g} / \mathrm{ml}$ leupeptin, $5 \mu \mathrm{g} / \mathrm{ml}$ aprotinin, $3 \mu \mathrm{M}$ benzamidine, $0.5 \mathrm{mM}$ DTT, $1 \mathrm{mM}$ PMSF) and was sonicated. The supernatant was taken after centrifugation at $12,000 \mathrm{rpm}$ for $10 \mathrm{~min}$ at $4{ }^{\circ} \mathrm{C}$. Protein $(15 \mu \mathrm{g})$ was used for SDS-polyacrylamide gel electrophoresis and immunoblotting with anti-phospho-Erk1/2 antibody (1:4000, Cell Signaling), anti-Cdc2 antibody, anti-phospho-vimentin antibody $(1: 2,000, \mathrm{MBL})$ that binds specifically to phosphorylated vimentin (serine 55) by Cdc2 kinase [18], and horseradish peroxidase (HRP)-conjugated secondary antibodies (1:1000; goat anti-rabbit; Santa Cruz, or sheep anti-mouse; Amersham Biosciences, Buckinghamshire, UK). Densitometric analysis of protein bands in the X-ray film was determined using the i-Solution software (Image \& Microscope Technology). 


\section{Immunofluorescence staining}

Samples were fixed with $4 \%$ paraformaldehyde and $4 \%$ sucrose in PBS at room temperature for $40 \mathrm{~min}$, permeablized with $0.5 \%$ nonidet P-40 in PBS, and blocked with $2.5 \%$ horse serum and $2.5 \%$ bovine serum albumin for $16 \mathrm{~h}$ at room temperature. Staining procedure was performed by incubating with primary antibodies raised against Cdc2 (1:100, Santa Cruz Biotech.), vimentin (1:1,000, Chemicon, Temecula, USA), phospho-vimentin (1:400, MBL), NF-200 (1:400, Sigma), ßIII-tubulin (TUJ1, 1:400, Cavance), S100 $\beta$ (1:400, Santa Cruz Biotech.), phospho-Erk1/2 (1:400, Cell Signaling), and GAP-43 (1:400, Santa Cruz Biotech.) followed by fluorescein-goat antimouse (1:400, Molecular probes, Eugene, OR, USA) or rhodamine-goat anti-rabbit secondary antibodies (1:400, Invitrogen, Carlsbad, CA, USA) in $2.5 \%$ horse serum and $2.5 \%$ bovine serum albumin for $90 \mathrm{~min}$ at room temperature. Cellular nuclei were stained with $2.5 \mu \mathrm{g} / \mathrm{ml}$ of Hoechst dye 33258 (bis-benzimide; Sigma) for $10 \mathrm{~min}$ before the final washing with $0.1 \%$ Triton $\mathrm{X}-100$ in PBS, and the sections or cells were cover-slipped with gelatin mount medium. Samples were viewed with a Nikon fluorescence microscope, and the images were captured by Nikon camera. The merged images were produced using layer-blending mode options of the Adobe Photoshop. For quantitative analysis of image data, average signals from 4 nonconsecutive sections per each animal were compared among experimental groups.

\section{Hot plate test}

Sciatic nerves of Balb/c mice (male, 22-24 g) were exposed on the middle thigh and taxol $(1.25 \mathrm{mg} / \mathrm{kg})$ or equivalent volume of DMSO vehicle was focally injected into the sciatic nerve. Twenty four hours later, herbal decoction $(400 \mathrm{mg} / \mathrm{kg})$ or saline was administered orally and was supplemented on a daily basis for 4 more days. On day 7 after taxol injection, animals were subjected to thermal sensitivity test on a hot plate. Animals were adapted for $10 \mathrm{~min}$ on the surface on a hot plate adjusted to $30{ }^{\circ} \mathrm{C}$, and immediately after, were placed for $30 \mathrm{~s}$ on the plate adjusted to $50{ }^{\circ} \mathrm{C}$. The latency to the lifting response of the hind paw and the number of withdrawal frequency were determined by analyzing realtime images of animal movement which were captured by a digital camera.

\section{Statistical analysis}

Data were presented as mean \pm standard error of mean (SEM). The mean number data in individual groups were compared by the Student's $t$-test or oneway ANOVA followed by Tukey test post-hoc analysis (SPSS computer software version 21.0), and statistically significant differences were reported as ${ }^{*} P<0.05$, ** $P<0.01, * * * P<0.001$.

\section{Results}

To validate the chemical profile of herbal extracts, BGJTD and 5 subgroups, we carried out HPLC analysis for chemical ingredients of herbal drugs. BGJTD and 5 subgroup extracts showed the peak profiles in the chromatogram that coincide with those of standard chemical solutions (Fig. 1). Specifically, HPLC profiles of BGJTD identified puerarin in Pueraria lobate Ohwi, albiflorin in Paeonia lactiflora pall, paeoniflorin in Paeonia lactiflora pall, 5-HMF in Rehmannia glutinosa, and chlorogenic acid in Angelica gigas. Other ingradients in Ba to Be subgroup decoctions are indicated in Table 2.

To investigate whether taxol and BGJTD influence on structural integrity of the peripheral nerve, sciatic nerve axons were visualized by immunofluorescence staining using anti-NF-200 antibody as axon marker. Comparison among experimental groups showed that the mean length of individual axons in the longitudinal nerve sections were significantly decreased by taxol injection indicating their fragmentation and then improved by BGJTD administration (Fig. 2a, b). Given that taxol stabilizes mictotubule assembly, we further investigated axonal morphology by immunostaining with neuronspecific $\beta$-III tubulin antibody (TUJ-1). Staining pattern of the sciatic nerve axons, as seen by elongated fibers in DMSO vehicle control, was disintegrated by taxol injection, which was then largely recovered in its integrity by BGJTD administration (Fig. 2c). Examination of neurons in the DRG at lumbar level 5 , where the cell bodies of sciatic nerve sensory axons reside, showed structural disruption of the neurons by taxol injection into the nerve. However, in animals administered with BGJTD, some, but not all, of neurons showed the morphology that is similar to those in the control animals (arrows in Fig. 2d).

Next, we examined the effects of taxol and BGJTD treatments on the neurite outgrowth of cultured DRG neurons. Taxol injection significantly reduced neurite length, but cotreatment with BGJTD improved neurite extension similar to vehicle-treated control group (Fig. 3a). Examination of DRG neurons merged with Hoechst-stained nuclear images revealed that many of non-neuronal cell nuclei in the culture were highly colocalized with the neurite processes, which was observed most clearly in a group treated with taxol and BGJTD (arrows in Fig. 3b).

Given that taxol interferes dynamic regulation of microtubule assembly, Schwann cells in the nerve might be affected adversely by taxol. Some Schwann cells in the control group showed thin, elongated morphology having physical contact with other cells. After taxol treatment, cells became more spherical and smaller (Fig. 4a), but co-treatment with BGJTD reverted them into an elongated morphology, which was similarly observed in control Schwann cells. To determine the 

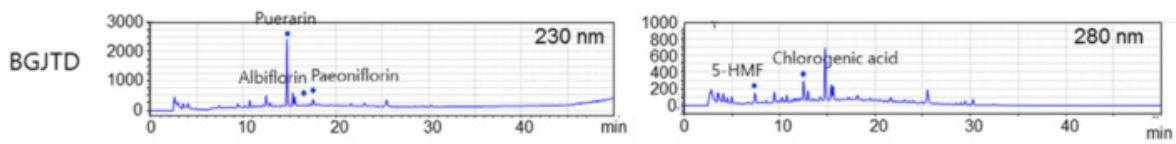

$\mathrm{Ba}$
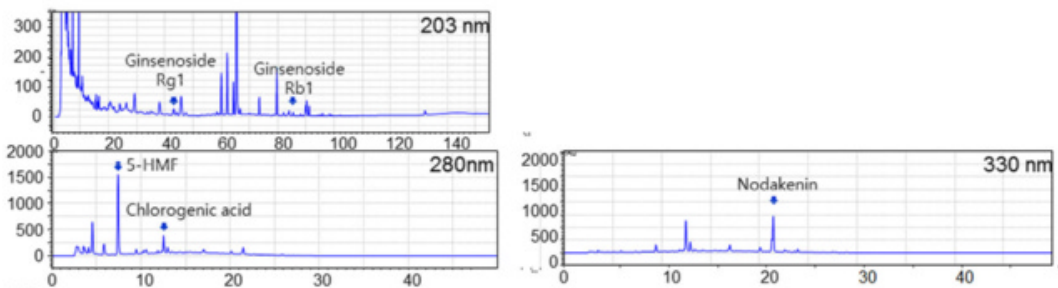

$\mathrm{BC}$

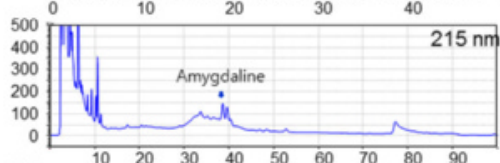

$\mathrm{Bd}$
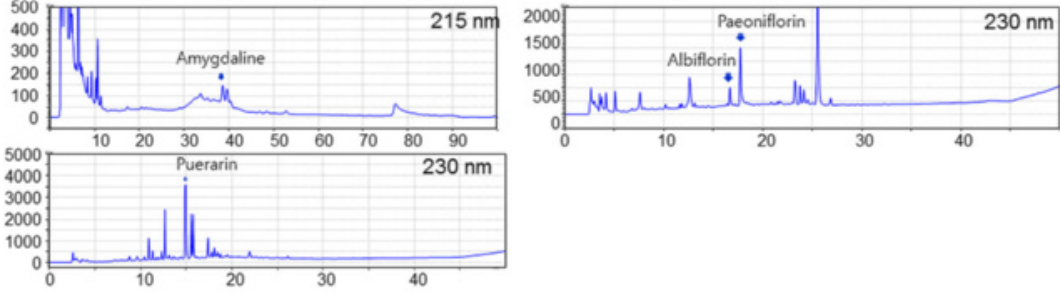

$\mathrm{Be}$
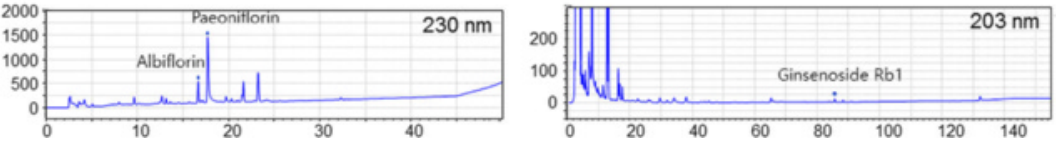

Fig. 1 HPLC profile of BGJTD and its subgroup extracts. Peaks that coincide with standard chemical solutions are marked by vertical arrows, and UV values applied to detect peaks are indicated in the figure

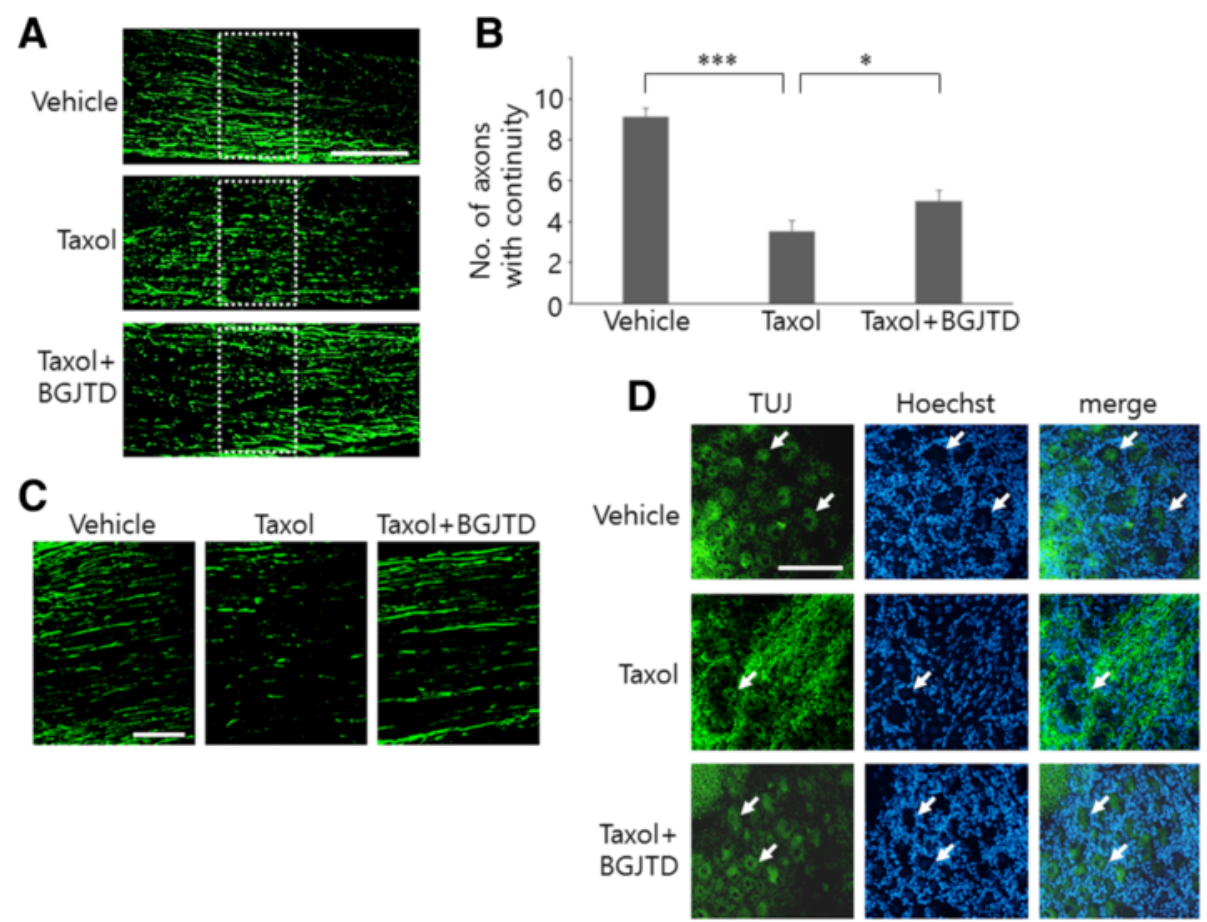

Fig. 2 Morphological changes of sciatic nerve axons and DRG neurons after taxol injection and BGJTD treatments. Sciatic nerve was exposed and treated with DMSO vehicle, taxol, or taxol plus BGJTD, and longitudinal sections of the nerve at the injected location (a, $\mathbf{c})$ and DRG at lumbar level 5 (d) were used for immunofluorescence staining for NF-200 and $\beta / 1$-tubulin. The number of axons whose length is longer than $100 \mu \mathrm{m}$, as illustrated with dotted rectangles in (a), was counted from the images, averaged from 3 nonconsecutive sections and compared among 3 experimental groups. Quantitative data are shown in (b). Soma DRG neurons was marked by arrows in (d). ${ }^{*} p<0.05,{ }^{* * *} p<0.001$ (One-way ANOVA, number of animals $=4)$. Scale bars in $(\mathbf{a}, \mathbf{c}, \mathbf{d}): 100 \mu \mathrm{m}$ 

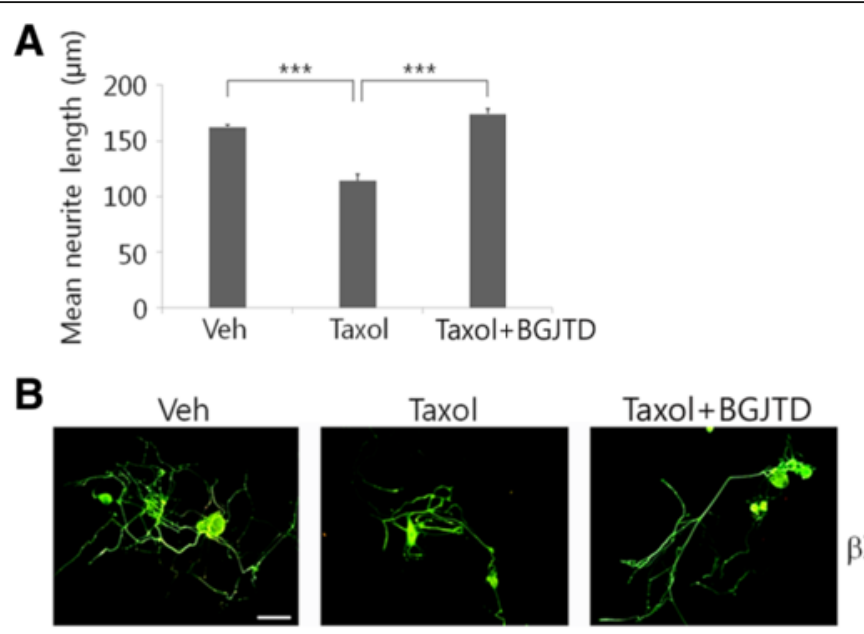

\section{$\beta$ III Tubulin}
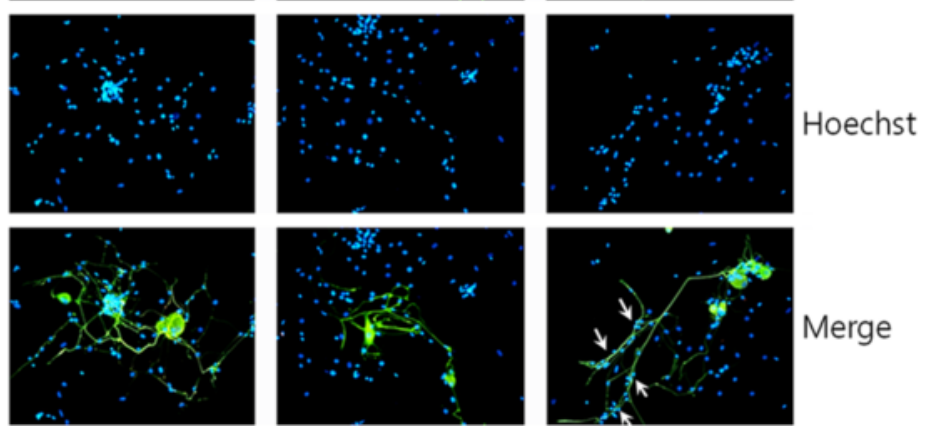

Merge

Fig. 3 Effects of BGJTD on the neurite outgrowth of DRG neurons. a Quantitative comparison of neurite outgrowth. $\mathbf{b}$ Representative images of DRG neurons co-cultured with Schwann cells. The neurites were visualized by immunostaining with anti- $\beta$ III tubulin antibody (green), and the distribution of co-cultured Schwann cells were identified by Hoechst nuclear staining (blue). In (a), neurite length was determined by analyzing more than 20 neurons in 7-10 random microscopic fields, averaged 4 independent experiments, and compared among experimental groups. Error bars denote standard error of mean (SEM). ${ }^{* *} p<0.001$ (One-way ANOVA, number of independent experiments $=4$ ). Scale bar: $50 \mu \mathrm{m}$

involvement of taxol and BGJTD in cell death regulation, levels of caspase 3 as a marker of apoptotic cell death were analyzed in cultured Schwann cells. Caspase 3 signals were clearly induced by taxol, but decreased to a large extent by BGJTD treatment (Fig. 4b, c).

To investigate that BGJTD and taxol treatments alter neural cell activity, we examined Erk1/2 activation in the sciatic nerve and DRG. Phospho-Erk1/2 levels was not altered by taxol alone, but increased by BGJTD administration (Fig. 5a). In the DRG, phospho-Erk1/2 levels were decreased by taxol, and then increased by BGJTD administration (Fig. 5b). As another indicator of nerve activation, we examined induction of Cdc2 kinase and its phosphorylation of vimentin intermediate filament protein as a substrate, which is known to be induced strongly but transiently from Schwann cells in the injured peripheral nerve $[19,20]$. Cdc2 was induced in the sciatic nerve by taxol injection, and BGJTD treatment upregulated Cdc2 with concomitant induction in phospho-vimentin (Fig. 5c). Immunohistochemical analysis for the sciatic nerve showed that both $\mathrm{Cdc} 2$ and phospho-vimentin were detected together in S100ß-labeled Schwann cells (Fig. 5d).
We further investigated whether BGJTD was beneficiary to axonal regeneration of the sciatic nerve after crush injury. Phospho-Erk1/2 levels were increased by crush injury and further upregulated by BGJTD treatment (Fig. 6a). Cdc2 was clearly induced in both proximal and distal portions of the sciatic nerves at day 7 after injury (Fig. 6b). Then the treatment of BGJTD suppressed Cdc2 production almost exclusively in the proximal stump while further increasing in the distal stump. Phospho-vimentin was weakly detected in the injured sciatic nerves but greatly increased in the distal portion of the nerve after BGJTD treatment. Immunofluorescence staining of the nerve fibers revealed that axonal elongation to the distal portion of the nerve was restricted to a certain extent when measured 3 days after injury; however, axon extension was much improved by BGJTD treatment (Fig. 6c). For quantitative analysis of axon regeneration by BGJTD, DRG neurons were retrogradely labeled by fluorescence dye DiI. DiIlabeled neurons, as an indicator of regenerating sensory axons, were clearly observed in many of DRG neurons, and the total number of labeled neurons was significantly higher in BGJTD-treated group than control group (Fig. 6d). 


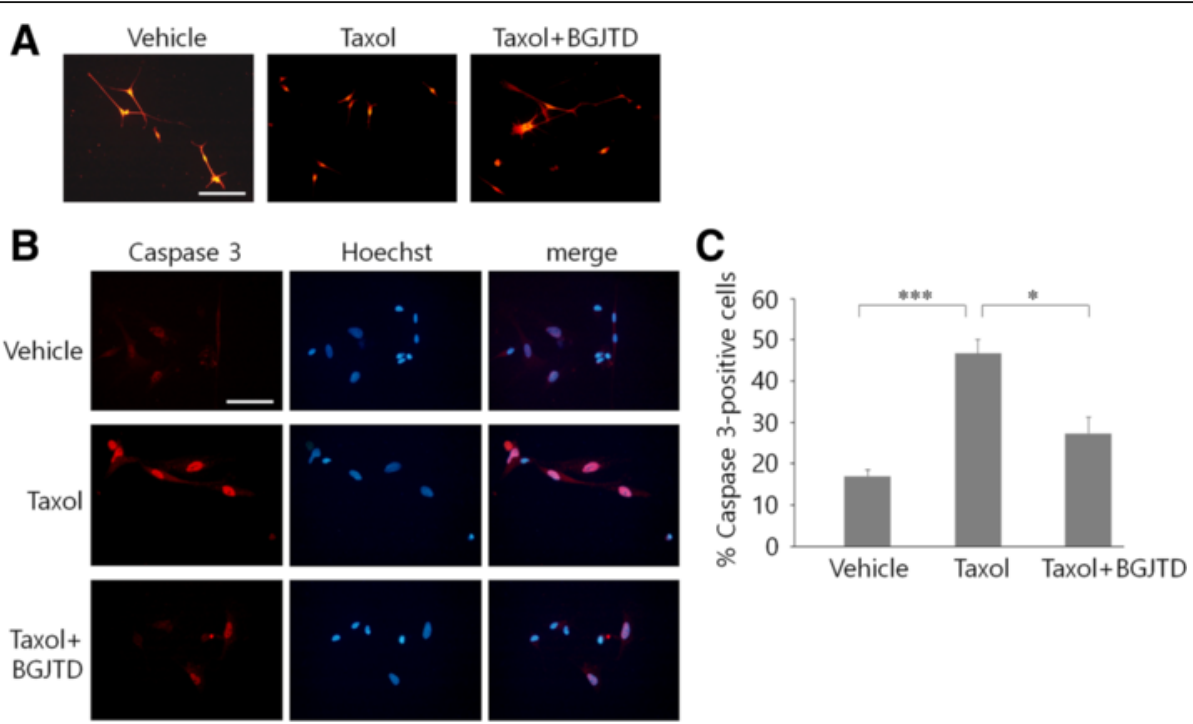

Fig. 4 Regulation of Schwann cell survival by BGJTD treatment. Schwann cells prepared from intact sciatic nerve were treated with DMSO vehicle, taxol, and taxol plus BGJTD. Fixed cells were used for immunostaining with anti-S100 $\beta$ antibody (a) and anti-caspase 3 antibody (b). Cells were also stained with Hoechst33258 to visualize individual nuclei (blue). a Morphological comparison of S100ß-stained Schwann cells after different treatments. b Immunofluorescence staining of caspase 3 in cultured Schwann cells. c Quantitative comparison of caspase 3-positive cells in cultured cells after different treatments. A percentage of caspase 3-positive cells from randomly selected microscopic fields (more than 5 fields) were determined from each experiment. ${ }^{*} p<0.05$, ${ }^{* *} p<0.001$ (One-way ANOVA, number of independent experiments $=4$ ). Scale bars in $(\mathbf{a}-\mathbf{c}): 100 \mu m$

BGJTD is a decoction composed of as many as 18 different herbal drugs. As an initial step to sort out the active components in its efficacy on the nerve repair, we divided them into 4 different groups (i.e., $\mathrm{Ba}, \mathrm{Bb}, \mathrm{Bc}$, and $\mathrm{Bd}$ ) based on the description of the traditional medicine (Table 2). After treating individual herbal drugs to cultured DRG neurons, we selected the most effective herbal drug from each group in inducing neurite outgrowth, and prepared the fifth herbal drug group, termed 'Be' (Table 2) (manuscript in preparation). We then treated each decoction to taxol-treated DRG neurons, and compared the neurite outgrowth. In vivo taxol injection suppressed neurite outgrowth and abrogated Schwann cell survival to a large extent as indicated by Hoechst nuclear staining (Fig. 7a, b). While the recovering effects of drug treatment on neurite outgrowth were variable, Be decoction was the most effective to enhance neurite outgrowth. It was also noted that the number of Hoechst-stained nuclei in Be was higher than other drugs and the elongated neurite was in close contact with many nuclei particularly at the tip (marked with a dotted circle in the enlarged image in Fig. 7b). In Be group, GAP-43 protein signals were not only observed in the cell body as other groups, but also were seen clearly in the elongating neurites. We further investigated the behavioral effect of BGJTD and Be extract on heat sensitivity. Withdrawal frequency and latency time in lifting the hind legs from the hot surface were significantly impaired by taxol administration, but were improved by BGJTD or Be treatment (Fig. 8a, b).

\section{Discussion}

The objective of the present study was to investigate whether BGJTD was effective for protecting the peripheral nerves from damage caused by taxol injection and physical injury. Taxol generated structural disruption of the nerve, and hampered Schwann cell survival adversely. Morphological and biochemical analyses of the sciatic nerve and DRG tissue revealed that taxol and crush injury generated nerve damage in a similar way. Importantly, these pathological injuries were largely recovered by BGJTD treatment. By sorting out the herbal components of BGJTD in terms of their activity to enhance neurite outgrowth, we selected the active herbal components, and a decoction Be from selected drugs was as effective as BGJTD in facilitating neurite outgrowth and responding to heat stimulation.

Since the initial discovery and pharmacological characterization, taxol has been used as a chemotherapeutic drug. However, together with several other anticancer drugs such as platinum compounds, taxanes, vinka alkaloids, bortezomib, and thalidomide, a large portion of patients given taxol therapy showed neuropathy accompanying severe pain [21, 22]. Considering stabilization effect of taxol on microtubule assembly, axonal cytoskeletal structures mainly composed of microtubule assembly might be damaged by taxol therapy. Studies with animals and in vitro culture showed that taxol caused inhibition of neurite outgrowth of cultured neurons, disrupted myelin structure, and retarded conduction of action potential [7-9]. 


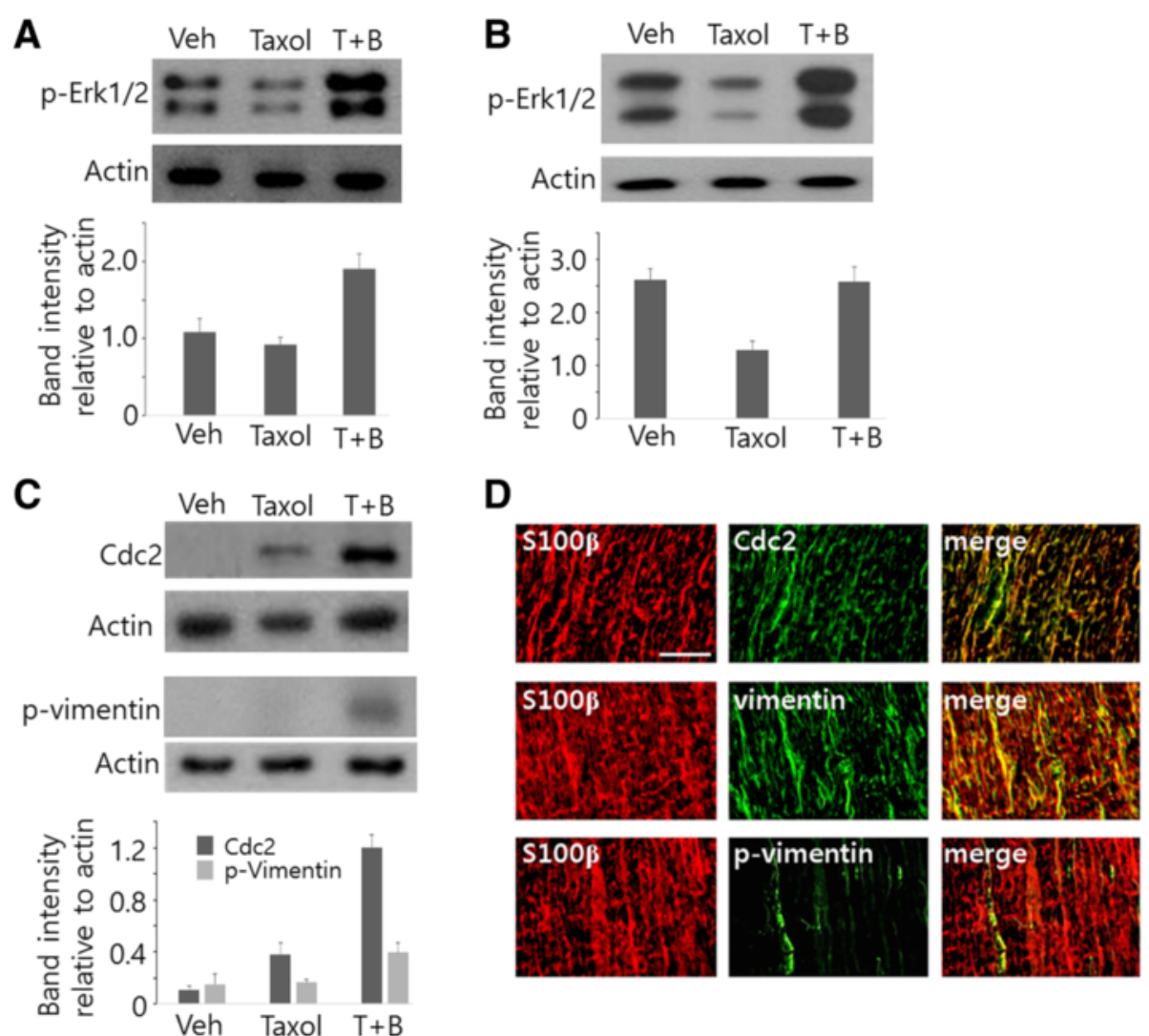

Fig. 5 Effects of taxol and BGJTD treatments on Erk1/2 and Cdc2 activity in the sciatic nerve. Western blot analysis of phospho-Erk $1 / 2$ in the sciatic nerves (a) and in the DRG (b) after taxol injection and BGJTD treatments ( $T+B)$. c Western blot analysis of Cdc2 and phospho-vimentin in the sciatic nerves treated with DMSO vehicle, taxol, and taxol plus BGJTD. $\mathbf{d}$ Representative images showing Cdc2 and phospho-vimentin signals in S100 $\beta$ labeled Schwann cells. Longitudinal sections from distal portion of the nerve treated with taxol and BGJTD were used in this experiment. Western blotting images in $\mathbf{( a - c}$ ) are the representatives from 3 independent experiments. Quantitative comparison of mean band intensity for target proteins relative to actin control in western blotting are shown in lower panels in (a-c). Scale bars in (d): $100 \mu \mathrm{m}$

After peripheral nerve injury, neuropathy can occur during repair process $[2,23]$. BGJTD has been used for the treatment of neuropathic pain and regulation of blood glucose levels in diabetic patients in the clinical traditional medicine, implicating the possible ameliorating effects of BGJTD on diabetic or anticancer-related neuropathy $[11,12]$. To understand biological basis of BGJTD action, we performed experiment using two models of peripheral nerve injury, and reached following conclusions; first, we found that taxol injection not only inhibited neurite outgrowth of cultured DRG neurons but also induced morphological abnormalities resulting in apoptotic death of primary Schwann cells. Taxol may have a detrimental effect on axonal regrowth by inhibiting Schwann cell interaction with axons [24]. Furthermore, functional degeneration of Schwann cells by taxol injection may prevent axon myelination and lead to morphometric alterations in action potential propagation $[25,26]$. Secondly, direct effects of taxol on the sciatic nerve axons were most clearly seen by immunohistochemical analysis in which BIII tubulin-labeled axons were largely degenerated by taxol injection. The structure of $\beta$ III tubulin-labeled DRG neurons at lumbar 5 was severely disintegrated by taxol injection, and phospho-Erk1/2 signals in DRG neurons were largely decreased by taxol. Previous studies have shown that phospho-Erk $1 / 2$ is induced in the peripheral nerve after injury, and can be transported retrogradely into the cell body where it leads to expression of target protein involved in axonal repair [27-29]. Here, taxol that may cause structural derangement from axon to soma could disrupt retrograde transport of Erk1/2 signaling, as has been shown by retarded transport of tracer in taxoltreated nerve [30]. Finally, BGJTD administration produced regenerative responses in the peripheral nerve. Signal intensity of apoptotic marker protein caspase 3 in taxol-treated Schwann cells was reduced by BGJTD, and $\mathrm{Cdc} 2$ activity, which was induced in the sciatic nerve after taxol injection or crush injury, was upregulated in the distal portion of the nerve. Moreover, neurite extension in cultured DRG neurons and axonal staining of in vivo sciatic nerves were improved in the BGJTD-treated 


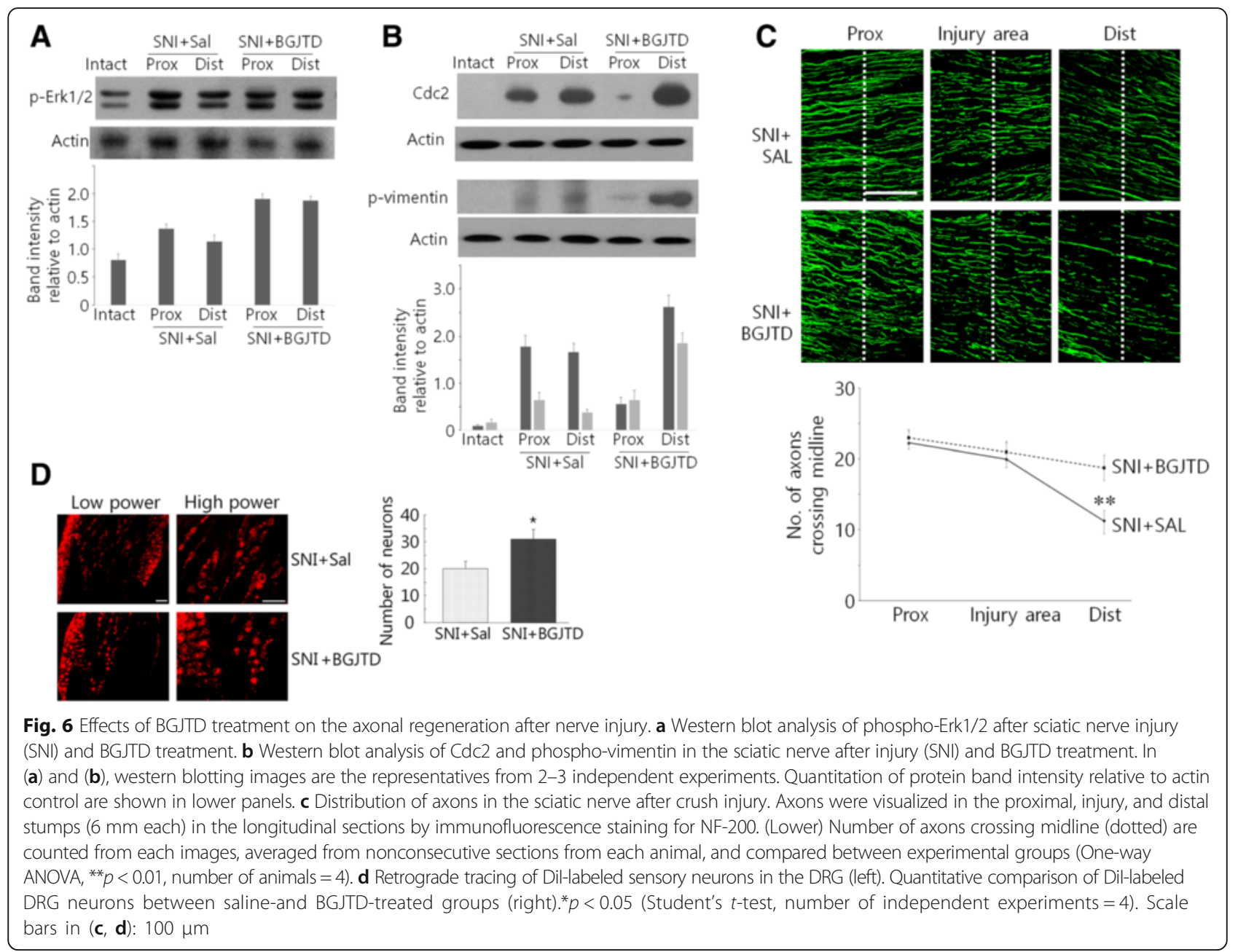

groups, compared to corresponding taxol-treated ones.

We have reported that vimentin phosphorylated by Cdc2 kinase is induced in Schwann cells from the injured nerve and involved in regenerative responses $[19,20]$. Here, Cdc2 and phospho-vimentin were detected from taxol-injected sciatic nerve, and interestingly, their levels were further upregulated by BGJTD treatment. Given that Cdc2 activation in Schwann cells is responsible for axonal regeneration, BGJTD-mediated Cdc2 induction in Schwann cells may facilitate repair process in the taxol-treated nerves. Indeed, neurite outgrowth in the taxol-treated DRG neurons was recovered by BGJTD to the level of control ones, and in vivo axon morphology, as identified by $\beta I I I-$ tubulin staining, was clearly improved by BGJTD. The protective effect of BGJTD was also shown in Schwann cells in which caspase 3 signals induced by taxol was largely decreased by BGJTD treatment. Thus, protective activity of BGJTD in both individual axons and Schwann cells may improve nerve repair, as demonstrated by increases in axon staining in the distal nerve stump and retrograde labeling of DRG sensory neurons (Fig. 6).

Our data showing the induction of $\mathrm{Cdc} 2$ and phospho-vimentin levels by BGJTD suggest possible molecular basis on how BGJTD acts on neuropathy. The BGJTD treatment upregulated Cdc2 levels and its phosphorylation of vimentin in taxol-injected nerves and the distal stump in the injured nerve. It was previously reported that Schwann cell activation in terms of Cdc2vimentin pathway is beneficiary to promote axonal regeneration of the peripheral nerve [20]. After peripheral nerve injury, axons in the distal stump degenerate and regrow from the proximal end, and in this process, Schwann cells are actively involved in eliminating degenerating debris and guiding the growth cones at the front [24]. Molecular factors that are expressed from activated Schwann cells including growth factors and antiinflammatory cytokines may play a role in alleviating peripheral nerve neuropathies [31, 32]. Timely interaction of Schwann cells with axons is important for recovering distal portion of injured nerve with minimizing 


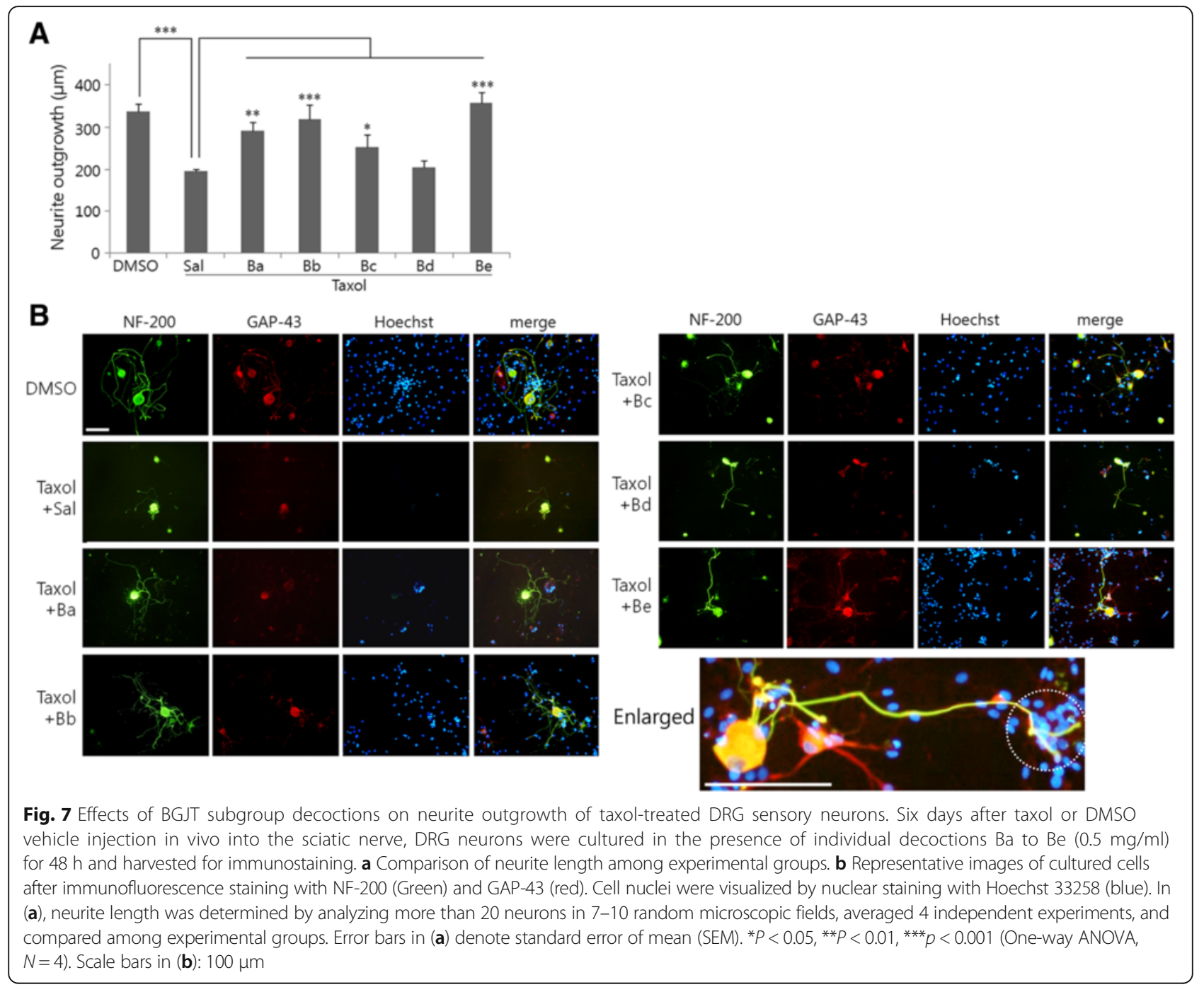

inflammation [33]. It was reported that activation of Cdc2-vimentin pathway in Schwann cells was linked to activation of membrane intergrin, which is further involved in intercellular communication with regrowing axons $[20,24]$. Whether BGJTD therapy is functionally associated with Schwann cell communication via integrin remains to be determined.

BGJTD is a decoction composed of 18 different herbal drugs and has been used in Asian medicine to treat neuropathy caused by diabetes and anticancer therapy
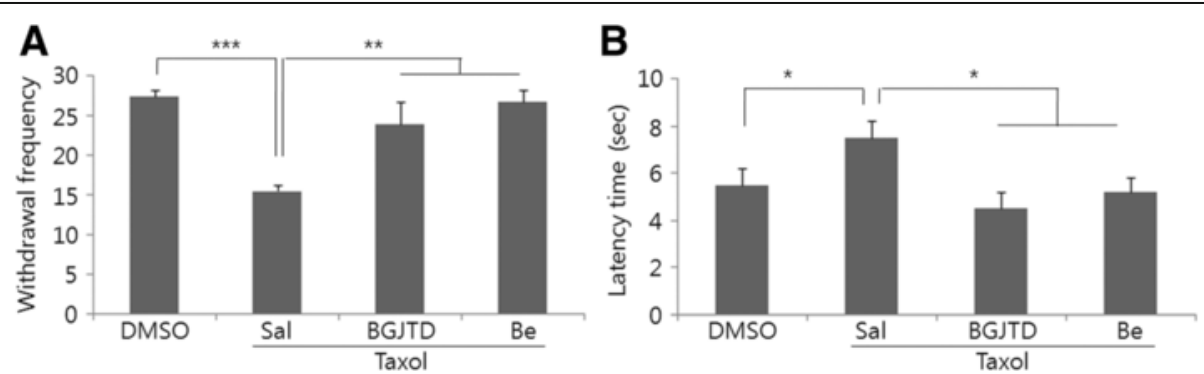

Fig. 8 Effects of taxol, BGJTD, and Be drugs on the heat sensitivity. Mice were given taxol followed by oral administration of BGJTD or Be, and were subjected to measure the lifting frequency of the hind paws (a) and the latency period after placing on the hot plate (b). Error bars in (a) denote standard error of mean (SEM). ${ }^{*} P<0.05,{ }^{* *} P<0.01,{ }^{* * *} p<0.001$ (One-way ANOVA, number of animals $=4$ ) 
[11]. A decoction shengmai san, which is used for the treatment of cardiovascular disorders in traditional medicine, shares the herbal component Ginseng radix with BGJTD and was reported to be involved in promoting regenerative responses after spinal cord injury [34]. Ginsenoside components such as Rg1 and Re were reported to facilitate axonal regeneration after peripheral nerve injury, or neuronal survival [35, 36], and ginsenoside $\operatorname{Re}$ was shown to activate Schwann cells in the injured nerve thereby promoting axonal regeneration [37]. However, given that BGJTD contains diverse herbal components, it is difficult to determine whether the efficacy of BGJTD is related to combinatorial activity or due to some specific components. Here, as an initial step to screen the possible active herbal components, we categorized 18 individual drugs into 4 groups based on description of the traditional medicine, and selected the most active components inducing neurite outgrowth of cultured neurons; a similar procedure was applied previously to search herbal drugs for therapeutic application to neurodegenerative diseases [38]. Our data showed that Be decoction composed of 4 active herbal drugs is as effective as BGJTD in inducing heat sensitivity of the hind paws. Further studies are critical to determine whether Be extract is comparable to BGJTD in regulating regenerative responses in association with peripheral nerve neuropathy.

\section{Conclusions}

In conclusion, our data showed that BGJTD, which is used clinically in the traditional Asian medicine, has a protective effect on neuropathic insults such as taxol injection or crush injury. By using in vitro screening procedure, we have selected some active herbal components, of which decoction displayed the efficacy of reinducing the optimal levels of heat sensitivity in taxol-treated animals. Accordingly, our data provide the biological basis of BGJDT on the treatment of the peripheral nerve neuropathy, and further implicate that some of the traditional medicinal descriptions on the efficacy of the herbal drugs may be applicable to the scientific approaches to identifying the active ingredients for the purpose of the therapeutic strategy.

\section{Abbreviations}

BGJTD: Bogijetong decoction; SNI: Sciatic nerve injury; DRG: Dorsal root ganglion; Cdc2: Cell division cycle 2; GAP-43: Growth-associated protein 43

\section{Acknowledgements}

The authors would like to thank all members in the Laboratory of Neurophysiology at Daejeon University.

\section{Funding}

This research was supported support by a grant of the Korea Health Technology R\&D Project through the Korea Health Industry Development Institute (KHIDI), funded by the Ministry of Health \& Welfare, Republic of Korea (grant number : H114C0963).
Availability of data and materials

All data and materials are contained and described within the manuscript.

\section{Authors' contributions}

SHA prepared BGJTD and carried out in vivo analysis experiments. SHA and CSC categorized BGJTD into 4 subgroup for experimental analysis. IAC and KJK performed cell culture and western blot experiments. CJK, UN, and CSC conceived of the study and discussed the data. SHA, UN, and CSC drafted and wrote the manuscript. All authors read and approved the final manuscript.

\section{Competing interests}

The authors declare that they have no completing interests.

\section{Consent for publication}

This information is not relevant.

\section{Ethics approval}

All procedures for experimental animal use were in strict accordance with the $\mathrm{NIH}$ guide for the care and use of laboratory animals and approved by the Committee on Use of Live Animals for Teaching and Research at

Daejeon University (Daejeon, Korea).

Received: 15 January 2016 Accepted: 14 October 2016

Published online: 22 October 2016

References

1. Feldman EL. Oxidative stress and diabetic neuropathy: a new understanding of an old problem. J Clin Invest. 2003;111:431-3.

2. Scholz J, Woolf CJ. The neuropathic pain triad: neurons, immune cells and glia. Nat Neurosci. 2007;10:1361-8.

3. Schiff PB, Horwitz SB. Taxol stabilizes microtubules in mouse fibroblast cells. Proc Natl Acad Sci U S A. 1980;77:1561-5.

4. Rowinsky EK, Donehower RC. The clinical pharmacology of paclitaxel (Taxol). Semin Oncol. 1993;20(4 Suppl 3):16-25.

5. Chaudhry V, Rowinsky EK, Sartorius SE, Donehower RC, Cornblath DR Peripheral neuropathy from taxol and cisplatin combination chemotherapy: clinical and electrophysiological studies. Ann Neurol. 1994:35:304-11.

6. Cavaletti G, Bogliun G, Crespi V, Marzorati L, Zincone A, Marzola M, et al. Neurotoxicity and ototoxicity of cisplatin plus paclitaxel in comparison to cisplatin plus cyclophosphamide in patients with epithelial ovarian cancer. J Clin Oncol. 1997;15:199-206.

7. Peters CM, Jimenez-Andrade JM, Kuskowski MA, Ghilardi JR, Mantyh PW. An evolving cellular pathology occurs in dorsal root ganglia, peripheral nerve and spinal cord following intravenous administration of paclitaxel in the rat. Brain Res. 2007;1168:46-59.

8. Cavaletti G, Tredici G, Braga M, Tazzari S. Experimental peripheral neuropathy induced in adult rats by repeated intraperitoneal administration of taxol. Exp Neurol. 1995:133:64-72.

9. Carozzi VA, Canta A, Oggioni N, Sala B, Chiorazzi A, Meregalli C, et al. Neurophysiological and neuropathological characterization of new murine models of chemotherapy-induced chronic peripheral neuropathies. Exp Neurol. 2010;226:301-9.

10. Hellal F, Hurtado A, Ruschel J, Flynn KC, Laskowski CJ, Umlauf M, et al. Microtubule stabilization reduces scarring and causes axon regeneration after spinal cord injury. Science. 2011;331:928-31.

11. Cho JH, Kim JM, Kim JH, Oh YS, Kim CJ. A case report of chemotherapyinduced neuropathic pain treated with oriental medicine. J Korean Orient Med. 2010;31:58-63.

12. Kim JM, Cho CS, Kim CJ. Clinical study of 8 diabetic patients with paresthesia. Kor J Ori Internal Med. 2010;31:184-91.

13. Lee JB, Kim CJ, Cho CS. Effects of Bogijetong-tang on diabetic-peripheral neuropathy induced by streptozotocin in the mouse. J Korean Med. 2013;34:126-42

14. Park SW, Kim CJ, Cho CS. Effects of nerve regeneration by Bogijetong-tang on peripheral nerves damaged by taxol and crush injury. Kor J Orient Int Med. 2013:34:384-404.

15. Röyttä M, Horwitz SB, Raine CS. Taxol-induced neuropathy: short-term effects of local injection. J Neurocytol. 1984;13:685-701.

16. Ertürk A, Hellal F, Enes J, Bradke F. Disorganized microtubules underlie the formation of retraction bulbs and the failure of axonal regeneration. J Neurosci. 2007;27:9169-80 
17. Seo TB, Han IS, Yoon JH, Hong KE, Yoon SJ, Namgung U, et al. Involvement of $\mathrm{Cdc} 2$ in axonal regeneration enhanced by exercise training in rats. Med Sci Sports Exerc. 2006;38:1267-76.

18. Tsujjmura K, Ogawara M, Takeuchi Y, Imajoh-Ohmi S, Ha MH, Inagaki M. Visualization and function of vimentin phosphorylation by cdc2 kinase during mitosis. J Biol Chem. 1994;269:31097-106.

19. Han IS, Seo TB, Kim KH, Yoon JH, Yoon SJ, Namgung U. Cdc2-mediated Schwann cell migration during peripheral nerve regeneration. J Cell Sci. 2007;120:246-55.

20. Chang IA, Oh MJ, Kim MH, Park SK, Kim BG, Namgung U. Vimentin phosphorylation by Cdc2 in Schwann cell controls axon growth via $\beta 1$ integrin activation. FASEB J. 2012;26:2401-13.

21. Loprinzi CL, Reeves BN, Dakhil SR, Sloan JA, Wolf SL, Burger KN, et al. Natural history of paclitaxel-associated acute pain syndrome: prospective cohort study NCCTG N08C1. J Clin Oncol. 2011:29:1472-8.

22. Quasthoff S, Hartung HP. Chemotherapy-induced peripheral neuropathy J Neurol. 2002;249:9-17.

23. Ide C. Peripheral nerve regeneration. Neurosci Res. 1996;25:101-21.

24. Namgung $U$. The role of Schwann cell-axon interaction $n$ peripheral nerve regeneration. Cells Tissues Organs. 2014;200:6-12.

25. Weiss MD, Luciano CA, Quarles RH. Nerve conduction abnormalities in aging mice deficient for myelin-associated glycoprotein. Muscle Nerve. 2001;24:1380-7.

26. Scherer SS. The biology and pathobiology of Schwann cells. Curr Opin Neurol. 1997;10:386-97.

27. Tsuda Y, Kanje M, Dahlin LB. Axonal outgrowth is associated with increased ERK 1/2 activation but decreased caspase 3 linked cell death in Schwann cells after immediate nerve repair in rats. BMC Neurosci. 2011;12:12.

28. Reynolds AJ, Hendry IA, Bartlett SE. Anterograde and retrograde transport of active extracellular signal-related kinase 1 (ERK1) in the ligated rat sciatic nerve. Neuroscience. 2001;105:761-71.

29. Segal RA, Greenberg ME. Intracellular signaling pathways activated by neurotrophic factors. Annu Rev Neurosci. 1996:19:463-89.

30. Alessandri-Haber N, Dina OA, Joseph EK, Reichling DB, Levine JD. Interaction of transient receptor potential vanilloid 4, integrin, and SRC tyrosine kinase in mechanical hyperalgesia. J Neurosci. 2008;28:1046-57.

31. Chen ZL, Yu WM, Strickland S. Peripheral regeneration. Annu Rev Neurosci. 2007;30:209-33.

32. Lehmann $\mathrm{HC}$, Höke A. Schwann cells as a therapeutic target for peripheral neuropathies. CNS Neurol Disord Drug Targets. 2010;9:801-6.

33. Scheib J, Höke A. Advances in peripheral nerve regeneration. Nat Rev Neurol. 2013:9:668-76.

34. Seo TB, Baek K, Kwon KB, Lee SI, Lim JS, Seol IC, et al. Shengmai-sanmediated enhancement of regenerative responses of spinal cord axons after injury in rats. J Pharmacol Sci. 2009;110:483-92.

35. Ma J, Li W, Tian R, Lei W. Ginsenoside Rg1 promotes peripheral nerve regeneration in rat model of nerve crush injury. Neurosci Lett. 2010:478:66-71.

36. Cheung ZH, So KF, Lu Q, Yip HK, Wu W, Shan JJ, Pang PK, Chen CF. Enhanced survival and regeneration of axotomized retinal ganglion cells by a mixture of herbal extracts. J Neurotrauma. 2002;19:369-78.

37. Wang L, Yuan D, Zhang D, Zhang W, Liu C, Cheng H, et al. Ginsenoside Re promotes nerve regeneration by facilitating the proliferation, differentiation and migration of Schwann cells via the ERK-and JNK-dependent pathway in rat model of sciatic nerve crush injury. Cell Mol Neurobiol. 2015;35:827-40.

38. Tohda C, Kuboyama T, Komatsu K. Search for natural products related to regeneration of the neuronal network. Neurosignals. 2005;14:34-45.

\section{Submit your next manuscript to BioMed Central and we will help you at every step:}

- We accept pre-submission inquiries

- Our selector tool helps you to find the most relevant journal

- We provide round the clock customer support

- Convenient online submission

- Thorough peer review

- Inclusion in PubMed and all major indexing services

- Maximum visibility for your research

Submit your manuscript at www.biomedcentral.com/submit
Biomed Central 